\title{
Reversal of Ampicillin Resistance in MRSA via Inhibition of Penicillin-Binding Protein 2a by Acalypha wilkesiana
}

\author{
Carolina Santiago, Ee Leen Pang, Kuan-Hon Lim, Hwei-San Loh, and Kang Nee Ting \\ Faculty of Science, University of Nottingham Malaysia Campus, Jalan Broga, 43500 Semenyih, Selangor, Malaysia \\ Correspondence should be addressed to Kang Nee Ting; kang-nee.ting@nottingham.edu.my
}

Received 26 February 2014; Revised 4 June 2014; Accepted 18 June 2014; Published 30 June 2014

Academic Editor: Ranadhir Chakraborty

Copyright (C) 2014 Carolina Santiago et al. This is an open access article distributed under the Creative Commons Attribution License, which permits unrestricted use, distribution, and reproduction in any medium, provided the original work is properly cited.

The inhibitory activity of a semipure fraction from the plant, Acalypha wilkesiana assigned as 9EA-FC-B, alone and in combination with ampicillin, was studied against methicillin-resistant Staphylococcus aureus (MRSA). In addition, effects of the combination treatment on PBP2a expression were investigated. Microdilution assay was used to determine the minimal inhibitory concentrations (MIC). Synergistic effects of 9EA-FC-B with ampicillin were determined using the fractional inhibitory concentration (FIC) index and kinetic growth curve assay. Western blot experiments were carried out to study the PBP2a expression in treated MRSA cultures. The results showed a synergistic effect between ampicillin and 9EA-FC-B treatment with the lowest FIC index of 0.19 (synergism $\leq 0.5$ ). The presence of 9EA-FC-B reduced the MIC of ampicillin from 50 to $1.56 \mu \mathrm{g} \mathrm{mL}^{-1}$. When ampicillin and 9EA-FC-B were combined at subinhibitory level, the kinetic growth curves were suppressed. The antibacterial effect of 9EA-FC-B and ampicillin was shown to be synergistic. The synergism is due the ability of 9EA-FC-B to suppress the activity of PBP2a, thus restoring the susceptibility of MRSA to ampicillin. Corilagin was postulated to be the constituent responsible for the synergistic activity showed by 9EA-FC-B.

\section{Introduction}

Infection caused by methicillin-resistance Staphylococcus aureus (MRSA) is a world-wide health problem. These infections are predominantly observed among immunocompromised patients in hospitals. However, in recent years, there have been an increasing number of fatalities, failed treatment, and healthcare costs. Progressive escalation of antibiotic resistance in MRSA has resulted in a limited option for treatment [1]. Thus, there is an immediate need for alternative therapies to control the spread of illness caused by MRSA.

A key strategy in combating a resistant microorganism is to suppress its resistance factor. There are at least two mechanisms that Staphylococci can evade beta-lactam toxicity which are by synthesizing the penicillin-binding protein $2 \mathrm{a}$ (PBP2a) and $\beta$-lactamases [2]. In normal circumstances, Staphylococcus aureus strains produce penicillin-binding proteins (PBPs) for synthesis of bacterial cell wall. However, when exposed to lethal concentration of beta-lactams antibiotics, resistant S. aureus strains produce PBP2a, which has unusually low binding affinity to beta-lactams [3]. PBP2a, in turn, replaces the function of normal PBPs (blocked by beta-lactams) in the resistant strains for cell wall biosynthesis [4]. This confers MRSA resistance to the entire beta-lactam family. Hence, suppression of PBP2a production is a promising approach to overcome MRSA's resistance. By doing so, there is a possibility to restore the susceptibility of MRSA to betalactam antibiotics [5].

It has been observed that extracts, fractions, or metabolites of plant origin are able to inhibit production of PBP2a when they are used in combination with current available antibiotics. In a review article [6], combinations of drugs such as beta-lactams and beta-lactamase inhibitors of plant origin have been shown to exhibit synergistic activities against antibiotic resistant microorganisms. The article also highlights plants as a source of small molecule antibiotics and synergism observed in natural products with antibiotics against growth of bacteria, fungus, and mycobacteria. Using combination treatment as a strategy to tackle resistant microorganism has also demonstrated several successes at 
experimental level. For an example, combination of corilagin from the plant, Arctostaphylos uva-ursi, with oxacillin successfully inhibited production of PBP2a in MRSA. The MIC of oxacillin and other tested beta-lactams was reduced between 100-fold and 2000-fold, strongly suggesting that combination treatment can potentially be an alternative method to combat virulence of MRSA [7].

A. wilkesiana is a medicinal plant which has widely been utilized for treating bacterial and fungal infections [8]. In some cases, the plant is also used to treat malaria, gastrointestinal problems [9], and potentially cancers [10, 11]. Previously, we found anti-MRSA and other antibacterial activities in the ethyl acetate and ethanol extracts of $A$. wilkesiana [12]. In continuation to our earlier findings, we have now embarked to further investigate the effects of the semipure extracts of $A$. wilkesiana in the reversal of ampicillin resistance in MRSA.

\section{Methods}

2.1. Plant Extraction. The plant material was collected from Broga, Selangor, Malaysia (September, 2010). Voucher sample is deposited in the herbarium of Faculty of Science, University of Nottingham Malaysia Campus, and assigned as UNMC 9. The dried plant material $(3.6 \mathrm{~kg})$ consisting of the whole plant was subjected to sequential extraction using $n$-hexane, followed by ethyl acetate and finally $95 \%$ ethanol $[13,14]$.

2.2. Isolation of Bioactive Fraction 9EA-FC-B. The ethyl acetate extract of $A$. wilkesiana (9EA) was fractionated by using vacuum liquid chromatography (silica gel). The solvent system used for elution was $n$-hexane $(\mathrm{He})$ with increasing amount of chloroform $\left(\mathrm{CHCl}_{3}\right)$ and $\mathrm{CHCl}_{3}$ with increasing amount of methanol $(\mathrm{MeOH})\left[\mathrm{He} / \mathrm{CHCl}_{3}(1: 1)\right.$ $\rightarrow \mathrm{CHCl}_{3} \rightarrow \mathrm{CHCl}_{3} / \mathrm{MeOH}(97: 3 \mathrm{v} / \mathrm{v}) \rightarrow \mathrm{CHCl}_{3} / \mathrm{MeOH}$ $(95: 5 \mathrm{v} / \mathrm{v}) \rightarrow \mathrm{CHCl}_{3} / \mathrm{MeOH}(93: 7 \mathrm{v} / \mathrm{v}) \rightarrow \mathrm{CHCl}_{3} / \mathrm{MeOH}$ $\left.(90: 10 \mathrm{v} / \mathrm{v}) \rightarrow \mathrm{CHCl}_{3} / \mathrm{MeOH}(85: 15 \mathrm{v} / \mathrm{v})\right]$. Fractions obtained were further fractionated via preparative centrifugal thin layer chromatography (silica gel) using a similar solvent system. The semipurified fractions were then tested for antiMRSA activity.

2.3. Microorganism and Growth Conditions. Methicillin sensitive $S$. aureus ATCC 11632 (MSSA) was grown in tryptic soy broth (TSB) (Hi-Media, India) at $37^{\circ} \mathrm{C}$ for $24 \mathrm{~h}$ with a shaking mode of $220 \mathrm{rpm}$. Aliquot from this suspension was streaked on tryptic soy agar (TSA) (Hi-Media, India) and incubated at $37^{\circ} \mathrm{C}$ for another $24 \mathrm{~h}$. Two to four single colonies from the TSA plate were inoculated in $10 \mathrm{~mL}$ of Muller Hinton broth (MHB) (Hi-Media, India) and allowed to grow at $37^{\circ} \mathrm{C}$ until they reached exponential stage $\left(2 \times 10^{8} \mathrm{CFU} \mathrm{mL}^{-1}\right)$. The suspension was then used for microbroth dilution assay. MRSA ATCC 43300 was grown similarly except all the media used was supplemented with $2 \%$ sodium chloride $(\mathrm{NaCl})$ (Merck, Germany), and incubation temperature was at $35^{\circ} \mathrm{C}$. Bacterial stocks were kept at $-80^{\circ} \mathrm{C}$ in TSB added with $10 \%$ (v/v) glycerol (Sigma, USA).
2.4. Test Samples. The crude ethyl acetate extract of $A$. wilkesiana, 9EA, and a bioactive fraction derived from it 9EAFC-B (identified from previous experiments) were dissolved in dimethyl sulfoxide (DMSO) (Sigma, USA) at stock concentration of $100 \mathrm{mg} \mathrm{mL}^{-1}$. Further dilution was carried out using media, and the final concentration of DMSO in the media did not exceed $1 \%$. Our earlier study has reported the lack of solvent (DMSO) effect in the test samples [14]. Antibiotics for susceptibility testing were prepared at $10 \mathrm{mg} \mathrm{mL}^{-1}$ in sterile distilled water. Tested antibiotics were ampicilin (Amresco, USA), oxacillin (Discovery Fine Chemicals, UK), and methicillin (Sigma, USA).

2.5. Determination of MIC. MICs of antibiotics, crude extract 9EA, and the active fraction 9EA-FC-B against MRSA and MSSA were determined via microdilution assay with a 96well plate. Test samples were tested in a twofold serial dilution. Antibiotics were tested with concentrations ranging from 0.19 to $100 \mu \mathrm{g} \mathrm{mL}^{-1}$ and plant extract samples from 0.09 to $12 \mathrm{mg} \mathrm{mL}^{-1}$.

MSSA and MRSA broth cultures were grown in MHB and $\mathrm{MHB}+2 \% \mathrm{NaCl}$, respectively, until an exponential stage $(2 \times$ $10^{8} \mathrm{CFU} \mathrm{mL}^{-1}$ ) was reached (see Section 2.3). These broth cultures were diluted to correspond to final inoculums of $5 \times 10^{5} \mathrm{CFU} \mathrm{mL}^{-1}$ upon inoculation into each well containing twofold serial dilutions of test sample. Media used in the assay were $\mathrm{MHB}$ for MSSA and $\mathrm{MHB}+2 \% \mathrm{NaCl}$ for MRSA. In the final incubation step, plates were incubated for $24 \mathrm{~h}$ at $37^{\circ} \mathrm{C}$ for MSSA and at $35^{\circ} \mathrm{C}$ for MRSA. General guidelines for this experiment were obtained from Clinical and Laboratory Standards Institute 2007 [15] with recommendations adapted from several other studies [16-18].

\subsection{Synergistic Studies}

2.6.1. Growth Curves Assay. MRSA was grown in a 96-well plate in the presence of following subinhibitory concentrations of 9EA-FC-B; $1 / 4 \times \mathrm{MIC}\left(0.75 \mathrm{mg} \mathrm{mL}^{-1}\right), 1 / 8 \times \mathrm{MIC}$ $\left(0.38 \mathrm{mg} \mathrm{mL}^{-1}\right)$, and $1 / 16 \times \mathrm{MIC}\left(0.19 \mathrm{mg} \mathrm{mL}^{-1}\right)$ in combination with subinhibitory concentrations of ampicillin ranging from $1 / 2 \times$ MIC to $1 / 64 \times \operatorname{MIC}\left(25\right.$ to $0.78 \mu \mathrm{g} \mathrm{mL}^{-1}$ ) under aerobic condition. Cell growth was monitored by reading optical density (OD) values at $600 \mathrm{~nm}$ at indicated time points for $24 \mathrm{~h}$. Reading was monitored by using Varioskan Flash Multimode Reader (Thermo Scientific, USA).

2.6.2. FIC Index Interpretation. FIC index for the combination treatments in synergy growth curves assay was calculated. The formula used was FIC ampicillin = MIC of ampicillin in combination/MIC ampicillin alone, FIC plant extract $=$ MIC of plant extract in combination/MIC of plant extract alone, and FIC index = FIC ampicillin + FIC plant extract. The combination was defined as synergy if the FIC index was $\leq 0.5$, indifference was defined $>0.5$ but $\leq 4.0$, and antagonism was defined as when the FIC index was $>4$ [19]. 
2.7. Protein Extraction. MRSA was grown in $\mathrm{MHB}+2 \%$ $\mathrm{NaCl}$ in the presence of subinhibitory concentrations of 9EAFC-B until late exponential phase. The bacterial lysates were prepared in an extraction buffer containing Tris and EDTA, and culture supernatants were harvested via centrifugation $4340 \mathrm{~g}$ at $4^{\circ} \mathrm{C}$ for $10 \mathrm{~min}$. The collected pellets were then treated with $150 \mathrm{mg} \mathrm{mL}^{-1}$ lysozyme, DNase, and protein inhibitors cocktail before being subjected to $2 \mathrm{~h}$ incubation at $37^{\circ} \mathrm{C}$. To enhance cell disruption, 15 -minute sonication in ice-bucket was done. Following $15 \mathrm{~min}$ of centrifugation at $13850 \mathrm{~g}$, the pellets were obtained as the insoluble protein extracts that were harvested in elution buffer containing Tris, urea, and sodium dihydrogen phosphate. Protein concentrations were measured using Pierce $660 \mathrm{~nm}$ protein assay.

2.8. SDS-PAGE and Western Blot Assay. Extracted protein $\left(3 \mu \mathrm{g} \mathrm{mL}^{-1}\right)$ was stained with $4 \mathrm{X}$ lithium dodecyl sulphate (LDS) sample buffer and subjected to sodium dodecyl sulfate (SDS)-polyacrylamide (12\%) gel electrophoresis run at 120 V. Upon completion, the gel was stained in Coomassie Blue staining solution until a clear background was obtained for scanning with GS-800 calibrated densitometer (Bio-Rad, USA). In western blot analyses, electrophoresed gels were transferred to BioTrace NT nitrocellulose transfer membrane (Pall, USA). Membranes were incubated overnight at $4^{\circ} \mathrm{C}$ in gelatin from cold water fish skin (blocking agent) (Sigma, USA). The production of PBP2a from MRSA was detected by probing the membranes with mouse anti-PBP2a primary antibody (Denka Seiken, Japan) and antiglyceraldehyde 3-phosphate dehydrogenase (GAPDH) (Thermo Scientific, USA) with a dilution factor of $1: 10000$. The same membranes were hybridized with anti-mouse horseradish peroxidase-linked secondary antibody (Abcam, UK) diluted to $1: 10000$ to facilitate colorimetric detection with $3,3^{\prime}, 5,5^{\prime}$-tetramethylbenzidine (TMB) substrate (Nacalai Tesque, Japan). Assay response was recorded using GS800 calibrated densitometer (Bio-Rad, USA). Densitometric quantification of western blot images was done using Image J 1.38 programme (Windows version of NIH Image). Results were scored in percentage of expression (\%) normalized to GAPDH control.

\section{Results}

3.1. Anti-MRSA Activity of Antibiotics and A. wilkesiana Extract. The MIC values of ampicillin, crude extract of $A$. wilkesiana 9EA, and fraction 9EA-FC-B for MRSA ATCC 43300 and MSSA 11632 are presented in Table 1. The MIC value of ampicillin against MRSA notably confirmed the resistance of the studied strain, while MIC value of ampicillin against MSSA revealed the susceptibility of this strain to the antibiotic. Crude extract 9EA demonstrated antibacterial activity against both the resistant and sensitive strains tested with a lower MIC value observed for MSSA. The anti-MRSA activity of fraction 9EA-FC-B was fourfold more effective than crude extract 9EA.
TABLE 1: MIC values of ampicillin, crude extract 9EA, and fraction 9EA-FC-B against MRSA and MSSA.

\begin{tabular}{lccc}
\hline Strain & $\begin{array}{c}\text { MiC } \\
\left(\mu \mathrm{g} \mathrm{mL}^{-1}\right)\end{array}$ & $\begin{array}{c}\text { 9EA } \\
\left(\mathrm{mg} \mathrm{mL}^{-1}\right)\end{array}$ & $\begin{array}{c}\text { 9EA-FC-B } \\
\left(\mathrm{mg} \mathrm{mL}^{-1}\right)\end{array}$ \\
\hline MRSA & 50 & 12 & 3 \\
MSSA & 6.25 & 6 & 3 \\
\hline
\end{tabular}

Values represent triplicates of three independent experiments.

3.2. Synergistic Effects of $9 E A-F C-B$ with Ampicillin on MRSA Growth Curves. The synergistic effects of 9EA-FC-B with ampicillin at subinhibitory concentrations on the growth of MRSA are shown in Figure 1. The growth inhibitory effects shown by both ampicillin and 9EA-FC-B on MRSA are concentration dependent, while the curve for the untreated MRSA culture (control) showed an exponential growth. Suppression of MRSA growth was detected when the MRSA cultures were treated with $9 \mathrm{EA}-\mathrm{FC}-\mathrm{B}$ alone at $1 / 4 \times \mathrm{MIC}, 1 / 8 \times$ MIC and 1/16 $\times$ MIC (not shown in graph). The growth curves suggested enhanced growth inhibitory effects when MRSA was treated with ampicillin at subinhibitory concentrations in the presence of 9EA-FC-B also at subinhibitory concentrations. The inhibitory effect was observed at as low as 1/32 $\times$ MIC ampicillin in the presence of $1 / 4 \times$ MIC 9EA-FC-B. Similar inhibitory effect was also achieved in the presence of $1 / 8 \times$ MIC of 9 EA-FC-B in combination with $1 / 16 \times$ MIC ampicillin.

3.3. Synergistic Effects of 9EA-FC-B with Ampicillin Based on FIC Index. The FIC indices for the tested combinations are presented in Table 2. Synergistic effects were observed when 9EA-FC-B was introduced in the treatment at $1 / 4 \times \mathrm{MIC}$, $1 / 8 \times \mathrm{MIC}$, and $1 / 16 \times \mathrm{MIC}$ with ampicillin at subinhibitory concentrations $(1 / 4$ to $1 / 32 \times \mathrm{MIC})$. In the presence of $1 / 4$ $\times$ MIC 9EA-FC-B, synergistic effects were observed for the widest range of subinhibitory concentrations of ampicillin, and the range gradually decreased as the subinhibitory concentration of 9EA-FC-B was lowered.

Analysis of the FIC indices revealed the new MIC values of ampicillin in the presence of 9EA-FC-B at subinhibitory level (Table 3). 9EA-FC-B at subinhibitory concentrations has enhanced the activity of ampicillin by up to 32-fold against MRSA; for example, MIC of ampicillin alone is $50 \mu \mathrm{g} \mathrm{mL}^{-1}$, while the MIC of ampicillin in the presence of 9EA-FC-B at $1 / 4 \times \mathrm{MIC}$ is $1.56 \mu \mathrm{g} \mathrm{mL}^{-1}$. It is notable that the MIC values of ampicillin (for MRSA) in the presence of $1 / 4 \times$ MIC 9EA-FC$B$ and 1/8 $\times$ MIC 9EA-FC-B are lower than the MIC value of ampicillin for MSSA, that is, $6.25 \mu \mathrm{g} \mathrm{mL}^{-1}$.

3.4. Expression of PBP2a. Expression of PBP2a was detected at $76 \mathrm{kDa}$. The percentage of PBP2a expression in tested treatments is summarized in Figure 2. GAPDH which served as an internal control was detected in all treatments (results not shown). The presence of PBP2a band was detected for MRSA cultures that were grown at subinhibitory concentrations of ampicillin $(1 / 16 \times \mathrm{MIC}$ and $1 / 32 \times \mathrm{MIC})$ in western blot 

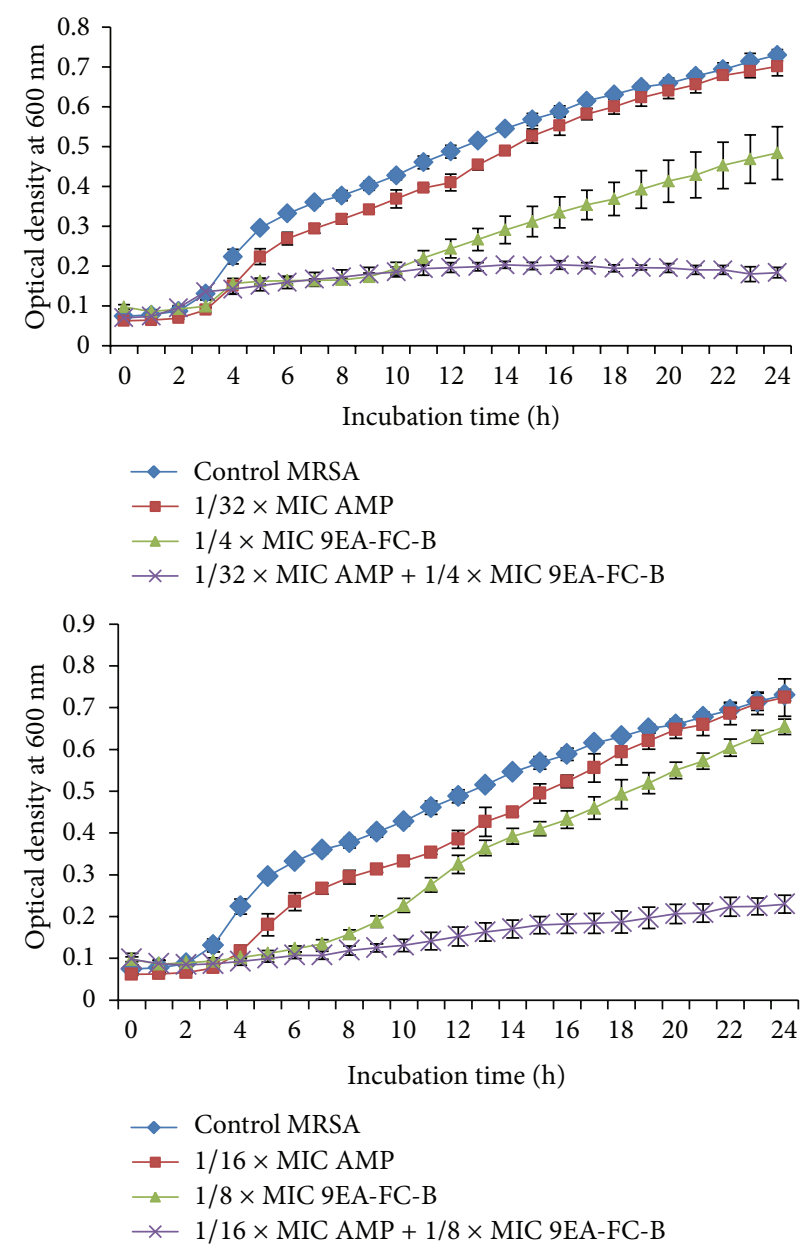

FIGURE 1: Effects of subinhibitory concentration of ampicillin alone, 9EA-FC-B alone and combination treatment on the growth of MRSA. Cell growth was measured by using OD at $600 \mathrm{~nm}$ at indicated time points. The curves represent triplicates of three independent experiments. Error bars show the standard deviation ( $\mathrm{AMP}=$ ampicillin, $\mathrm{MIC}=$ minimum inhibitory concentrations).

TABLE 2: FIC indices of some combinations of ampicillin and 9EA-FC-B for MRSA.

\begin{tabular}{lccc}
\hline Ampicillin $\left(\mu \mathrm{g} \mathrm{mL}^{-1}\right)$ & & 9 EA-FC-B $\left(\mathrm{mg} \mathrm{mL}^{-1}\right)$ \\
$1 / 8 \times \operatorname{MIC}(0.38)$ & 0.65 & 0.56 \\
\hline $1 / 2 \times$ MIC $(25)$ & 0.75 & 0.36 & 0.31 \\
$1 / 4 \times$ MIC $(12.5)$ & 0.43 & 0.25 & - \\
$1 / 8 \times$ MIC $(6.25)$ & 0.38 & 0.19 & - \\
$1 / 16 \times$ MIC $(3.125)$ & 0.31 & - & - \\
$1 / 32 \times$ MIC $(1.563)$ & 0.28 & - & - \\
$1 / 64 \times$ MIC $(0.781)$ & - & MIC $(0.75)$ & - \\
\hline
\end{tabular}

Values represent triplicates of three independent experiments. Index interpretation: $\leq 0.5=$ synergy, $>0.5$ but $\leq 4.0=$ indifference, and $>4=$ antagonism. $($ MIC $=$ minimum inhibitory concentrations; $-=$ no activity.)

experiment. Both western blot and quantitative densitometric analysis showed that these cultures have higher expression of PBP2a compared to the untreated MRSA culture (control). Inhibition of PBP2a expression was observed when MRSA cultures were exposed to subinhibitory concentrations of 9EA-FC-B, where no PBP2a band was seen at 1/4 × MIC 9EAFC-B and only a very low expression (3.9\%) was detected at $1 / 8 \times$ MIC 9EA-FC-B. Likewise, the MRSA culture did not show the presence of $\mathrm{PBP} 2 \mathrm{a}$ when grown in the presence of $1 / 32 \times$ MIC ampicillin $+1 / 4 \times$ MIC 9EA-FC-B.

\section{Discussion}

The scope of the study is to explore the potential of using active plant extracts or fractions to combat resistance in 


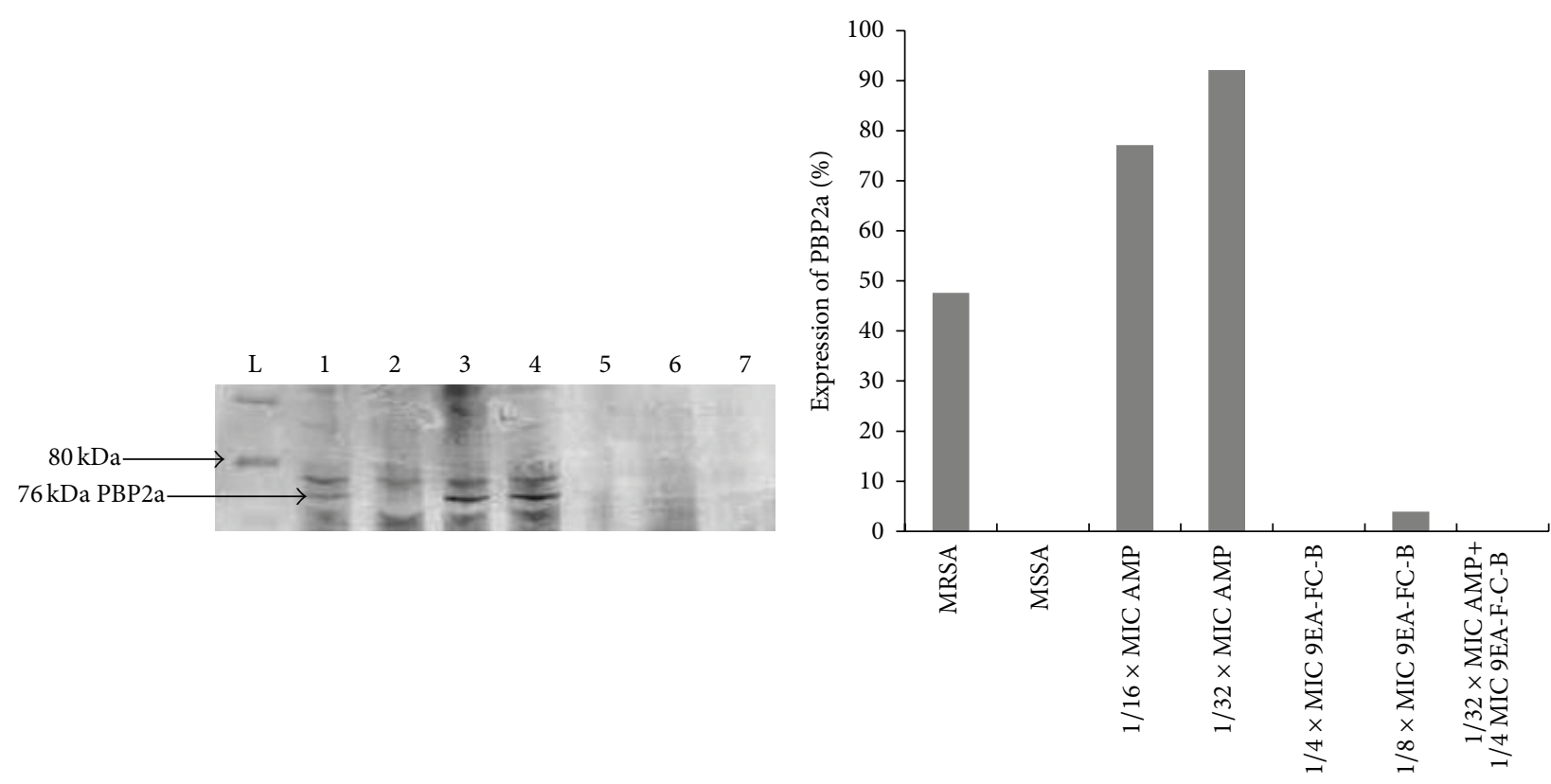

(a)

(b)

FIGURE 2: Expression of penicillin-binding protein 2a (PBP2a) of MRSA cultures grown in the presence of subinhibitory concentrations of 9EA-FC-B and ampicillin. (a) Western blot image, lane, L; molecular mass markers, 1: control MRSA, 2: control MSSA, 3: 1/16 $\times$ MIC AMP, 4: 1/32 $\times$ MIC AMP, 5: 1/4 × MIC 9EA-FC-B, 6: 1/8 $\times$ MIC 9EA-FC-B, 7: 1/32 $\times$ MIC AMP + 1/4 × MIC 9EA-FC-B. (b) Quantitative densitometric analysis of PBP2a expression of MRSA cultures grown in the presence of ampicillin alone, 9EA-FC-B alone, and in combinations, normalized to GAPDH loading control. (AMP = ampicillin; $\mathrm{MIC}=$ minimum inhibitory concentrations).

TABLE 3: MIC values of ampicillin in combination with subinhibitory concentrations of 9EA-FC-B for MRSA.

\begin{tabular}{lc}
\hline Treatment & MIC $\left.(\mu \mathrm{g} \mathrm{mL})^{-1}\right)$ of ampicillin \\
\hline Ampicillin alone & 50 \\
With 9EA-FC-B at $1 / 4 \times$ MIC & 1.56 \\
With 9EA-FC-B at $1 / 8 \times$ MIC & 3.13 \\
With 9EA-FC-B at $1 / 16 \times$ MIC & 12.5
\end{tabular}

Values represent triplicates of three independent experiments (MIC = minimum inhibitory concentrations).

MRSA. For the first time, in 2006, U.S. Food and Drug Administration (FDA 2006) has approved a special green tea extract containing a proprietary mixture of phytochemicals (the active ingredient listed as Polyphenon E) [20], as a prescription drug for the topical (external) treatment of genital warts caused by the human papilloma virus (HPV). This example reinforces the efforts to study medicinal plant extracts for possible application in clinical practice. The present result established the antimicrobial activity of fraction 9EA-FC-B obtained from the ethyl acetate crude extract of A. wilkesiana. It also demonstrated synergism between the fraction 9EA-FC-B and ampicillin in overcoming resistance of MRSA by inhibiting production of PBP2a. In the presence of subinhibitory concentrations of 9EA-FC-B, the MIC of ampicillin was reduced by as much as 32-fold, from $50 \mu \mathrm{g} \mathrm{mL}^{-1}$ to $1.56 \mu \mathrm{g} \mathrm{mL}^{-1}$, indicating that MRSA became more sensitive to ampicillin when fraction 9EA-FC-B was introduced in the treatment. Based on these findings, we predict that the active constituents from fraction 9EA-FC$B$ may potentially be used for combating MRSA's virulence. Several researches have exploited the synergistic effects of natural products for drug development [21-23]. The most apt example that is closely related to the present study is the synergistic effects of corilagin and tellimagrandin I in combination with beta-lactam antibiotics on antibacterial activity against MRSA by inactivation of PBP2a [24].

Results of bacterial growth curve experiment indicated that combination of 9EA-FC-B with ampicillin (both agents at subinhibitory concentrations) distinctly suppressed the growth of MRSA in contrast to MRSA cultures that were treated with either 9EA-FC-B or ampicillin alone. Generally, MIC of ampicillin reduced when subinhibitory concentration of 9EA-FC-B increased in the combination treatment. From the kinetic growth curves, we were able to deduce that the antimicrobial action of the ampicillin and 9EAFC-B combination was observed at the beginning of the exponential phase. A very minimal bacterial growth was seen with increase in incubation hours. Instead of growing rapidly during the exponential stage, the graph portrayed low growth of bacterial cells (approximately fourfold lower) with extended lag phase compared to the untreated MRSA (control). An extended lag phase was also detected for MRSA treated with 9EA-FC-B alone. Lag phase is the particular stage when bacteria equilibrate to adapt to the new environment by undergoing macromolecular repair and synthesis of cellular growth through DNA replications [25]. Hence, we deduced 
that a lengthy lag phase observed in our experiment is due to the inhibition of DNA replications that delays the cellular growth process. The prolonged lag phase of MRSA observed in this experiment is reminiscent of the action of fluoroquinolones that caused inhibition of DNA replication in MRSA, leading to a longer lag phase [26]. A potent antimicrobial action was also identified at the exponential phase in which bacterial cells were prevented from growing rapidly in the presence of 9EA-FC-B alone and in combination with ampicillin. This phenomenon showed probable interference in cell division which involves multiple rounds of DNA synthesis that are controlled by a variety of gene regulatory processes $[27,28]$. The plausible mechanism of action of 9EA-FC-B observed at the exponential phase is therefore associated with the interruption of cell division that causes membrane derangements and failure in membrane functions.

As for interpretation of the FIC indices, lower indices indicate better synergism [19]. Based on the FIC indices obtained, eight out of the 18 combinations tested showed synergistic effects. The presence of 9EA-FC-B significantly enhanced the potency of ampicillin by up to 32-fold (MIC reduced from $50 \mu \mathrm{g} \mathrm{mL}^{-1}$, in the absence of 9EA-FC-B, to $1.56 \mu \mathrm{g} \mathrm{mL}^{-1}$, in the presence of $1 / 4 \times$ MIC of 9EA-FC-B) for MRSA.

Ampicillin is a beta-lactam antibiotic that is designed to inhibit PBPs involved in late stage of peptidoglycan biosynthesis. Interference with peptidoglycan biosynthesis causes deformities in the bacterial cell wall and eventually leads to cell death due to high internal osmotic pressure. Nearly, all bacteria can be inhibited by interfering in mechanism of peptidoglycan synthesis [29]. Nevertheless, targeting this mechanism is no longer effective due to the production PBP2a in MRSA. The blocking of normal PBPs by beta-lactams did not exert effects on peptidoglycan or cell wall synthesis, because PBP2a replaces their function and ensures normal formation of cell wall in presence of lethal concentration of beta-lactam drugs [30]. However, in this study, we experimentally demonstrated restoration of ampicillin's antimicrobial activity by the addition of 9EA-FC$B$ at subinhibitory level.

The synergistic activity observed between 9EA-FC-B and ampicillin against MRSA was shown to be associated with inhibition of PBP2a. PBP2a is an inducible protein that regulates methicillin resistance. Its expression is heterogeneous in nature amidst level of resistance differing to the beta-lactam being used [31]. The mecA gene complex which encodes for this protein encompasses the regulatory genes, mecI and $m e c R$. Interaction of beta-lactam antibiotics with these regulatory genes eventually allows expression of $m e c A$ in terms of production of PBP2a [32-34]. As such, the occurrence of intense PBP2a bands in MRSA cultures grown in low concentrations of ampicillin compared to the control culture in western blot experiment suggested the induction of mecA gene transcription. In contrast, no PBP2a bands were detected in MRSA cultures that were exposed to $1 / 4 \times$ MIC of 9EA-FB-C, while PBP2a is only very mildly expressed when the concentration of 9EA-FC-B was lowered to $1 / 8 \times$ MIC. This suggests that 9EA-FC-B can either inhibit the production of PBP2a or directly inactivate PBP2a. This suggestion is in agreement with the observation that 9EAFC-B enhanced the ampicillin susceptibility of MRSA. In 2004, Shiota et al. [24] reported the antibacterial effect of corilagin and tellimagrandin I (isolated from Arctostaphylos uva-ursi and Rosa canina, resp.) against MRSA. The two polyphenolic compounds had exceptionally reduced MICs of beta-lactams for MRSA via inactivation of PBP2a. We believe that corilagin (a tannin) is also present in the test fraction 9EA-FC-B, since corilagin was previously isolated and identified by our colleagues from the same source of plant materials [35]. This is further supported by phytochemical testing that showed the presence of tannins in 9EA-FC-B (data not shown). Although Shimizu et al. [7] previously reported that corilagin enhanced antibacterial activity of various beta-lactams by 100- to 2000-fold against MRSA, 9EAFC-B was only shown to enhance the activity of ampicillin by up to 32 -fold in the present study. The stark difference in antibacterial enhancements can readily be rationalized by the fact that 9EA-FC-B is a semipure fraction that possibly contains only a small amount of corilagin in addition to many other secondary metabolites that were assumed to have negligible effect on the antibacterial activity observed.

\section{Conclusion}

From the results reported in this study, we found that the antibacterial effect of the fraction 9EA-FC-B obtained from A. wilkesiana and ampicillin is synergistic. The synergism is due the ability of 9EA-FC-B to suppress the production of PBP2a or directly inactivate it, leading to the restoration of the susceptibility of MRSA to ampicillin.

\section{Conflict of Interests}

The authors declared no conflict of interests.

\section{Acknowledgment}

This study was supported by Science Fund scheme under Ministry of Agriculture and Agro-Based Industry Malaysia (MOA), Malaysia, with Grant no. M0048.54.01.

\section{References}

[1] J. Snider, M. Rapids, and B. Rivard, "Methicillin-resistant Staphylococcus aureus: a review of current antibiotic therapy," Spectrum Health Grand Rapids, 2012.

[2] M. T. Gillespie, J. W. May, and R. A. Skurray, "Antibiotic resistance in Staphylococcus aureus isolated at an Australian hospital between 1946 and 1981," Journal of Medical Microbiology, vol. 19, no. 2, pp. 137-147, 1985.

[3] B. Berger-Bächi and S. Rohrer, "Factors influencing methicillin resistance in staphylococci," Archives of Microbiology, vol. 178, no. 3, pp. 165-171, 2002.

[4] B. Berger-Bächi, "Expression of resistance to methicillin," Trends in Microbiology, vol. 2, no. 10, pp. 389-393, 1994.

[5] J. Vouillamoz, J. M. Entenza, P. Hohl, and P. Moreillon, "LB11058, a new cephalosporin with high penicillin-binding protein $2 \mathrm{a}$ affinity and activity in experimental endocarditis due 
to homogeneously methicillin-resistant Staphylococcus aureus," Antimicrobial Agents and Chemotherapy, vol. 48, no. 11, pp. 4322-4327, 2004.

[6] S. Hemaiswarya, A. K. Kruthiventi, and M. Doble, "Synergism between natural products and antibiotics against infectious diseases," Phytomedicine, vol. 15, no. 8, pp. 639-652, 2008.

[7] M. Shimizu, S. Shiota, T. Mizushima et al., "Marked potentiation of activity of $\beta$-lactams against methicillin-resistant Staphylococcus aureus by corilagin," Antimicrobial Agents and Chemotherapy, vol. 45, no. 11, pp. 3198-3201, 2001.

[8] P. I. Alade and O. N. Irobi, "Antimicrobial activities of crude leaf extracts of Acalypha wilkesiana," Journal of Ethnopharmacology, vol. 39, no. 3, pp. 171-174, 1993.

[9] B. E. Akinde and O. O. Odeyemi, "Extraction and microbiological evaluation of the oils from the leaves of Acalypha wilkesiana," Nigerian Medical Journal, vol. 17, pp. 163-165, 1987.

[10] S. W. Lim, K. N. Ting, T. D. Bradshaw et al., "Acalypha wilkesiana extracts induce apoptosis by causing single strand and double strand DNA breaks," Journal of Ethnopharmacology, vol. 138, no. 2, pp. 616-623, 2011.

[11] S. W. Lim, H. S. Loh, K. N. Ting, T. D. Bradshaw, and N. A. Zeenathul, "Acalypha wilkesiana ethyl acetate extract enhances the in vitro cytotoxic effects of $\alpha$-tocopherol in human brain and lung cancer cells," International Journal of Bioscience, Biochemistry and Bioinformatics, vol. 3, no. 4, pp. 335-340, 2013.

[12] M. Othman, S. Genapathy, P. S. Liew et al., "Search for antibacterial agents from Malaysian rainforest and tropical plants," Natural Product Research, vol. 25, no. 19, pp. 1857-1864, 2011.

[13] G. Spigno and D. M. de Faveri, "Antioxidants from grape stalks and marc: influence of extraction procedure on yield, purity and antioxidant power of the extracts," Journal of Food Engineering, vol. 78, no. 3, pp. 793-801, 2007.

[14] M. Othman, H. S. Loh, C. Wiart, T. J. Khoo, K. H. Lim, and K. N. Ting, "Optimal methods for evaluating antimicrobial activities from plant extracts," Journal of Microbiological Methods, vol. 84, no. 2, pp. 161-166, 2011.

[15] Clinical and Laboratory Standards Institute (CLSI), "Laboratory methods for detection of methicillin-resistant Staphylococcus aureus (MRSA)," Supplement M100-S17, 2007.

[16] J. M. Andrews, "Determination of minimum inhibitory concentrations," Journal of Antimicrobial Chemotherapy, vol. 48, no. 1, pp. 5-16, 2001.

[17] D. F. J. Brown, "Detection of methicillin/oxacillin resistance in staphylococci," Journal of Antimicrobial Chemotherapy, vol. 48, no. 1, pp. 65-70, 2001.

[18] P. Cos, A. J. Vlietinck, D. V. Berghe, and L. Maes, “Anti-infective potential of natural products: how to develop a stronger in vitro proof-of-concept," Journal of Ethnopharmacology, vol. 106, no. 3, pp. 290-302, 2006.

[19] R. L. White, D. S. Burgess, M. Manduru, and J. A. Bosso, "Comparison of three different in vitro methods of detecting synergy: time-kill, checkerboard, and E test," Antimicrobial Agents and Chemotherapy, vol. 40, no. 8, pp. 1914-1918, 1996.

[20] U.S Food and Drug Administration (FDA), CDER New Molecular Entity (NME) Drug and New Biologic Approvals in Calendar Year, 2006.

[21] F. J. Schmitz, A. C. Fluit, M. Lückefahr et al., "The effect of reserpine, an inhibitor of multidrug efflux pumps, on the invitro activities of ciprofloxacin, sparfloxacin and moxifloxacin against clinical isolates of Staphylococcus aureus," Journal of Antimicrobial Chemotherapy, vol. 42, no. 6, pp. 807-810, 1998.
[22] F. R. Stermitz, P. Lorenz, J. N. Tawara, L. A. Zenewicz, and K. Lewis, "Synergy in a medicinal plant: Antimicrobial action of berberine potentiated by 5 '-methoxyhydnocarpin, a multidrug pump inhibitor," Proceedings of the National Academy of Sciences of the United States of America, vol. 97, no. 4, pp. 1433-1437, 2000.

[23] Y. Cho, N. L. Schiller, and K. Oh, "Antibacterial effects of green tea polyphenols on clinical isolates of methicillin-resistant Staphylococcus aureus," Current Microbiology, vol. 57, no. 6, pp. 542-546, 2008.

[24] S. Shiota, M. Shimizu, J. Sugiyama, Y. Morita, T. Mizushima, and T. Tsuchiya, "Mechanisms of action of corilagin and tellimagrandin I that remarkably potentiate the activity of $\beta$-lactams against methicillin-resistant Staphylococcus aureus," Microbiology and Immunology, vol. 48, no. 1, pp. 67-73, 2004.

[25] S. Dukan and T. Nyström, "Bacterial senescence: stasis results in increased and differential oxidation of cytoplasmic proteins leading to developmental induction of the heat shock regulon," Genes \& Development, vol. 12, no. 21, pp. 3431-3441, 1998.

[26] R. A. Venezia, B. E. Domaracki, A. M. Evans, K. E. Preston, and E. M. Graffunder, "Selection of high-level oxacillin resistance in heteroresistant Staphylococcus aureus by fluoroquinolone exposure," Journal of Antimicrobial Chemotherapy, vol. 48, no. 3, pp. 375-381, 2001.

[27] J. M. Navarro Llorens, A. Tormo, and E. Martínez-García, "Stationary phase in gram-negative bacteria," FEMS Microbiology Reviews, vol. 34, no. 4, pp. 476-495, 2010.

[28] M. D. Rolfe, C. J. Rice, S. Lucchini et al., "Lag phase is a distinct growth phase that prepares bacteria for exponential growth and involves transient metal accumulation," Journal of Bacteriology, vol. 194, no. 3, pp. 686-701, 2011.

[29] P. Lambert, "Mechanism of action of antibiotics and synthethic anti-infective agents," in Hugo and Russell's Pharmaceutical Microbiology, S. P. Denyer, N. A. Hodges, and S. P. Gorman, Eds., pp. 202-206, Blackwell Publishing, Oxford, UK, 7th edition, 2004.

[30] A. Smith, "Bacterial resistance to antibiotics," in Hugo and Russell's Pharmaceutical Microbiology, S. P. Denyer, N. A. Hodges, and S. P. Gorman, Eds., pp. 220-223, Blackwell, Oxford, UK, 7th edition, 2004.

[31] H. F. Chambers, "Methicillin resistance in staphylococci: molecular and biochemical basis and clinical implications," Clinical Microbiology Reviews, vol. 10, no. 4, pp. 781-791, 1997.

[32] G. L. Archer and J. M. Bosilevac, "Signaling antibiotic resistance in staphylococci," Science, vol. 291, no. 5510, pp. 1915-1916, 2001.

[33] H. Z. Zhang, C. J. Hackbarth, K. M. Chansky, and H. F. Chambers, "A proteolytic transmembrane signaling pathway and resistance to $\beta$-lactams in Staphylococci," Science, vol. 291, no. 5510, pp. 1962-1965, 2001.

[34] S. Deresinski, "Methicillin-resistant Staphylococcus aureus: an evolutionary, epidemiologic, and therapeutic odyssey," Clinical Infectious Diseases, vol. 40, no. 4, pp. 562-573, 2005.

[35] W. M. Din, J. Chu, G. Clarke et al., "Antioxidant and cytoprotective effects of an ethanol extract of Acalypha wilkesiana var. macafeana from Malaysia," Natural Product Communications, vol. 8, no. 3, pp. 375-380, 2013. 

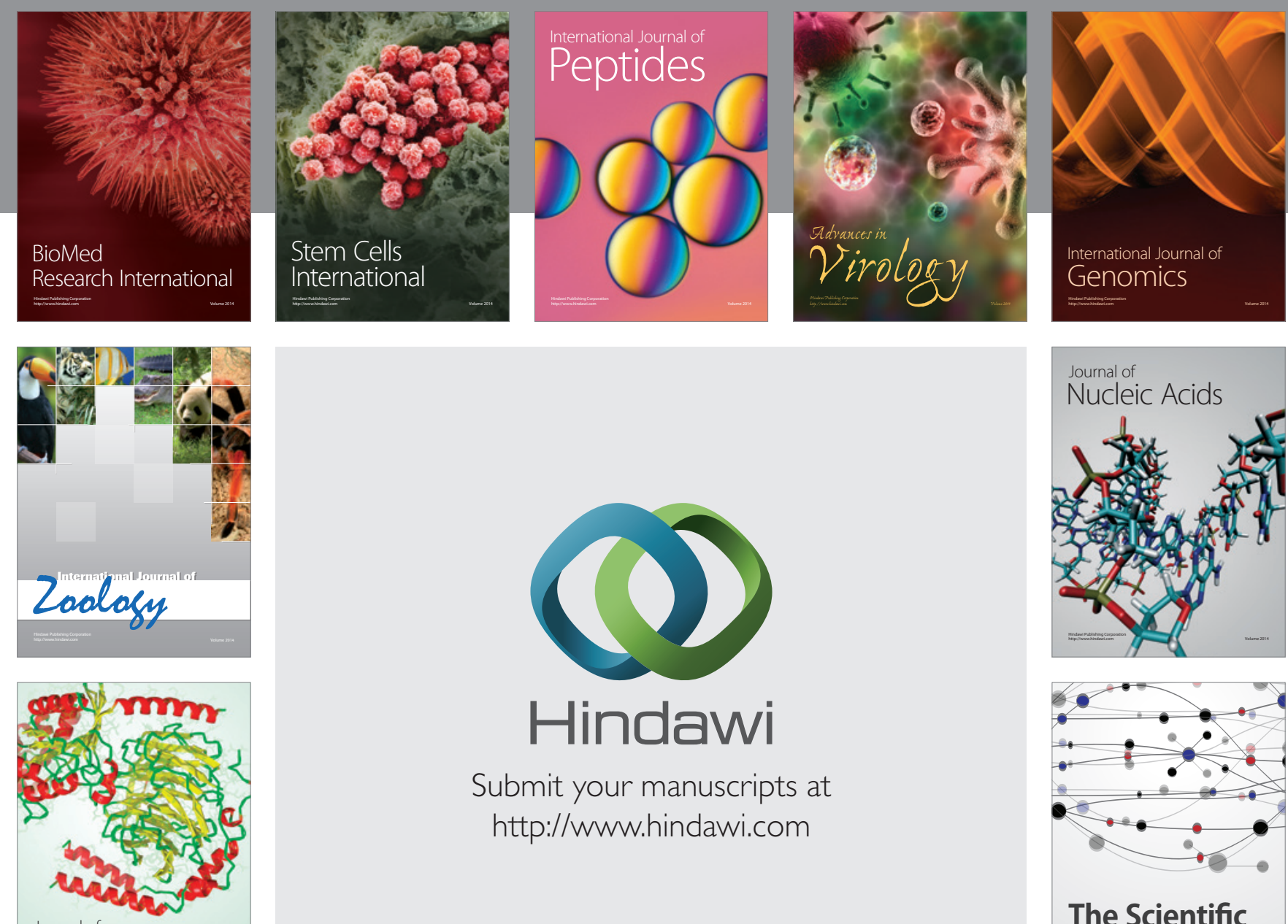

Submit your manuscripts at

http://www.hindawi.com

Journal of
Signal Transduction
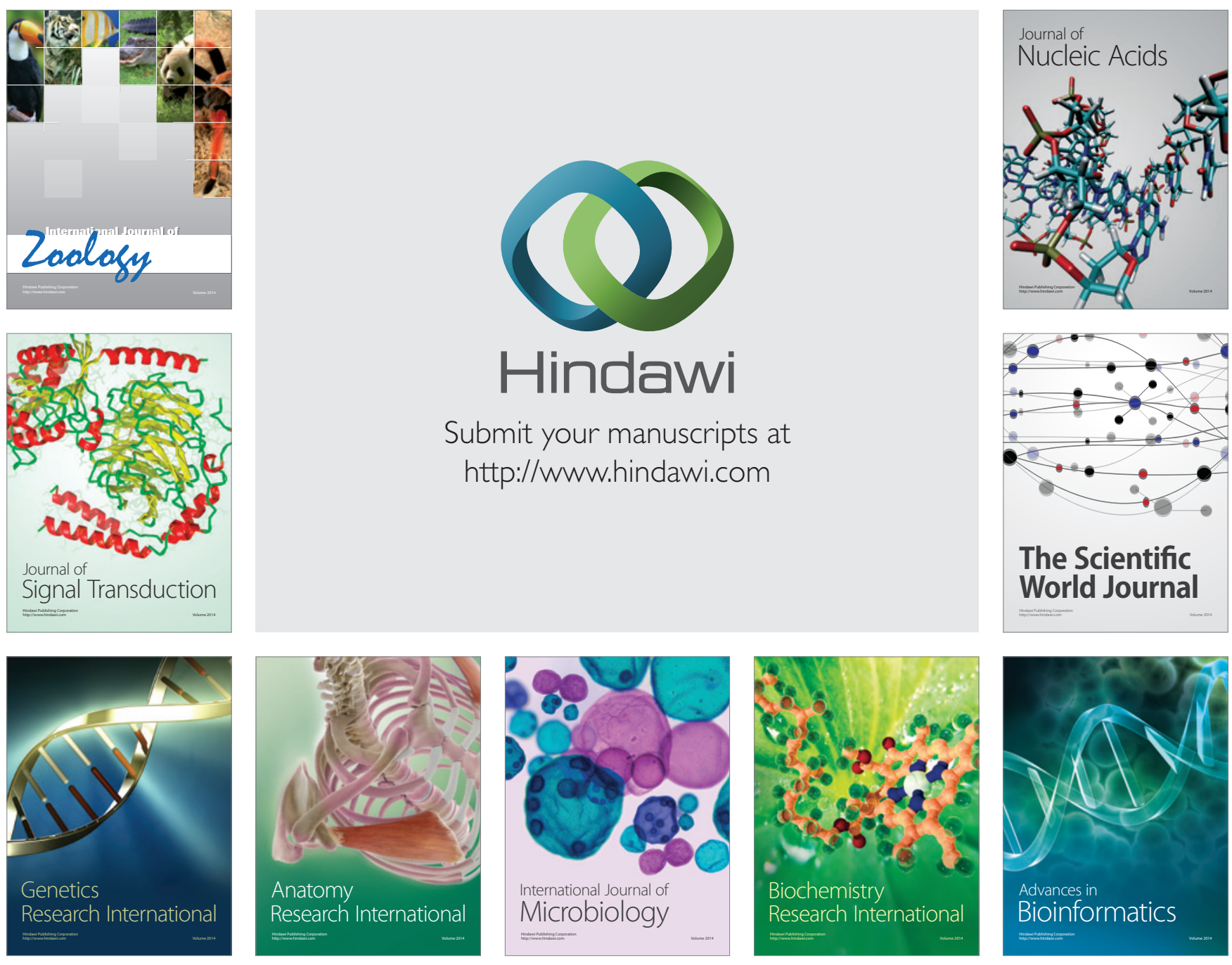

The Scientific World Journal
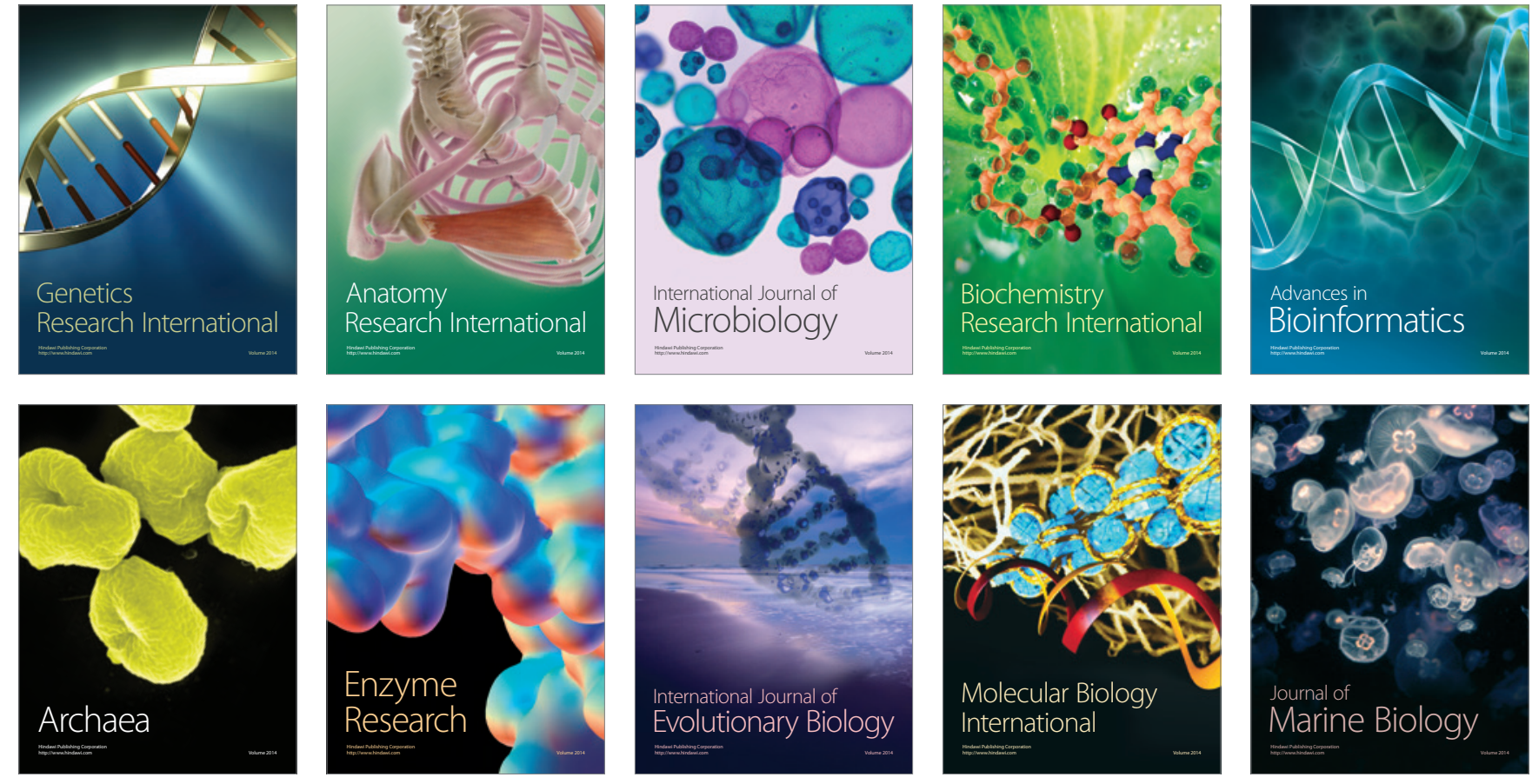